\title{
MATERIAL EFFECTS AND DETECTOR RESPONSE CORRECTIONS FOR BUNCH LENGTH MEASUREMENTS*
}

\author{
W. Zacherl, I. Blumenfeld, M. Berry, F.-J. Decker, M.J. Hogan, R. Ischebeck, R. Iverson, N. Kirby, \\ R. Siemann, D. Walz (SLAC, Menlo Park, CA), C.E. Clayton, C. Huang, C. Joshi, W. Lu, K.A. \\ Marsh, W.B. Mori, M. Zhou (UCLA, Los Angeles, CA), T.C. Katsouleas, P. Muggli, E. Oz (USC, \\ Los Angeles, CA).
}

\begin{abstract}
A typical diagnostic used to determine the bunch length of ultra-short electron bunches is the auto-correlation of coherent transition radiation. This technique can produce artificially short bunch length results due to the attenuation of low frequency radiation if corrections for the material properties of the Michelson interferometer and detector response are not made. Measurements were taken using FTIR spectroscopy to determine the absorption spectrum of various materials and the response of a Molectron P1-45 pyroelectric detector. The material absorption data will be presented and limitations on the detector calibration discussed.
\end{abstract}

\section{INTRODUCTION}

Simulations predict the minimum bunch length of the electron beam in the Final Focus Test Beam (FFTB) facility at the Stanford Linear Accelerator Center (SLAC) is on the order of 30 femtoseconds r.m.s. A Michaelson interferometer measures the bunch length by taking the autocorrelation of the Coherent Transition Radiation (CTR) emitted as the high energy beam $(28.5 \mathrm{GeV})$ passes through a $20 \mathrm{~mm}$ diameter, 1 micron-thick titanium foil. The radiation intensity is measured by a P1-45 pyroelectric detector. The resultant auto-correlation trace (ACT) is a compilation of approximately 1,000 electron bunch CTR measurements. The ACT is then corrected with a digital filter to remove material effects. Finally the full width at half maximum (FWHM) is calculated with a Lorentzian least squares fit. Application of this technique without correcting for material effects results in an artificially smaller bunch length.

To determine the most suitable materials to use as vacuum windows and beamsplitters, data for various materials was collected using a Bruker, IFS 66v/S, FTIR spectrometer. Various spectra are shown in figure 1a-1f. Both the standard DLATSGS detector and He cooled bolometer where used to collect data over the shown spectral range. The standard MIR globar was used as an IR source.

\section{RESULTS}

Pure silicon was determined to be the best material for both the vacuum window and beamsplitter, see figures $1 \mathrm{~d}$ and 1c respectively. As a vacuum window, silicon minimises the distortion of the CTR pulse due to its flat transmission curve through the desired spectral range,
$10 \mu \mathrm{m}-740 \mu \mathrm{m}$. As a beamsplitter, silicon has nearly equivalent transmission and reflection coefficients. This results in near maximal contributions from each branch of the interferometer. The amplitude from each branch is proportional to the product of the transmission and reflection coefficients of the beam splitter. See Figure 1 for a comparison of several common materials. Peaks exceeding unity in the transmission spectra between $13.6 \mu \mathrm{m}$ and $14 \mu \mathrm{m}$ are artefacts from the FTIR spectrometer. They are caused by resonances in the spectrometer's beamsplitter.

Figure 2 is the response of the P1-45 pyroelectric detector as a function of wavelength. The data was also collected with a Bruker FTIR. On the top is the raw, uncalibrated data. The peak response of the pyroelectric detector corresponds to the peak of a blackbody spectrum at 993K (also shown). In calculating the response curve on the bottom, the MIR globar used as the radiation source is assumed to have an emission spectrum equivalent to the depicted blackbody spectrum.

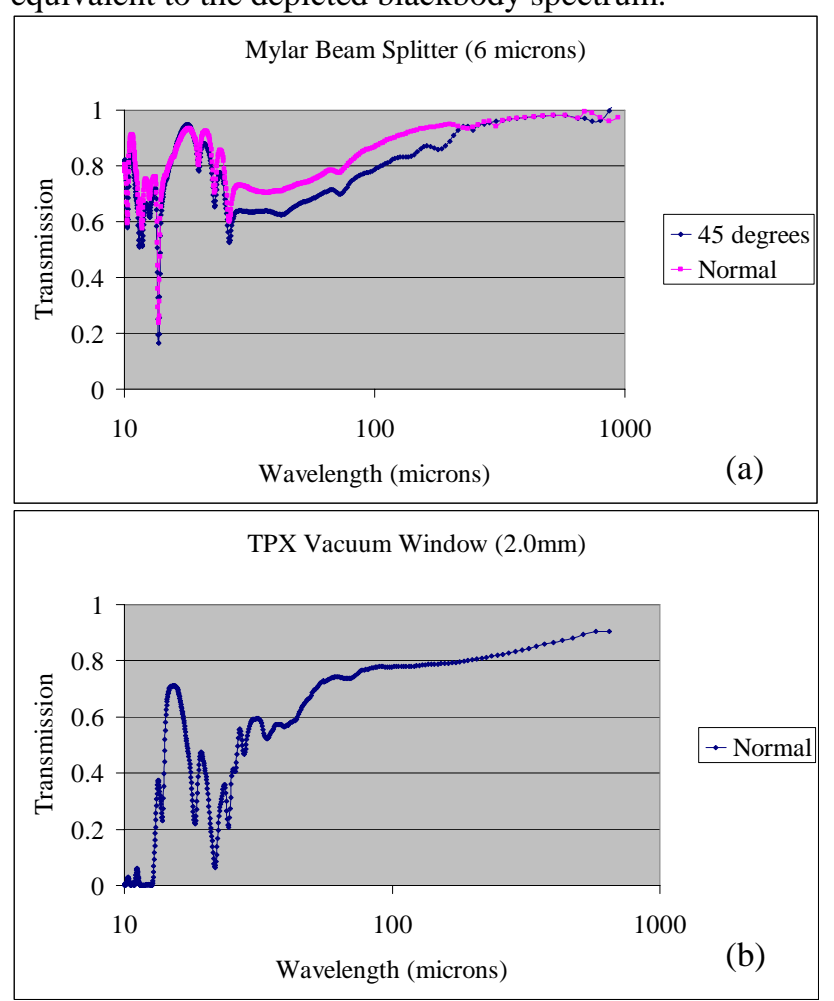

\footnotetext{
*Work supported by Department of Energy contract DE-AC02-

\#walter.zacherl@slac.stanford.edu
} 

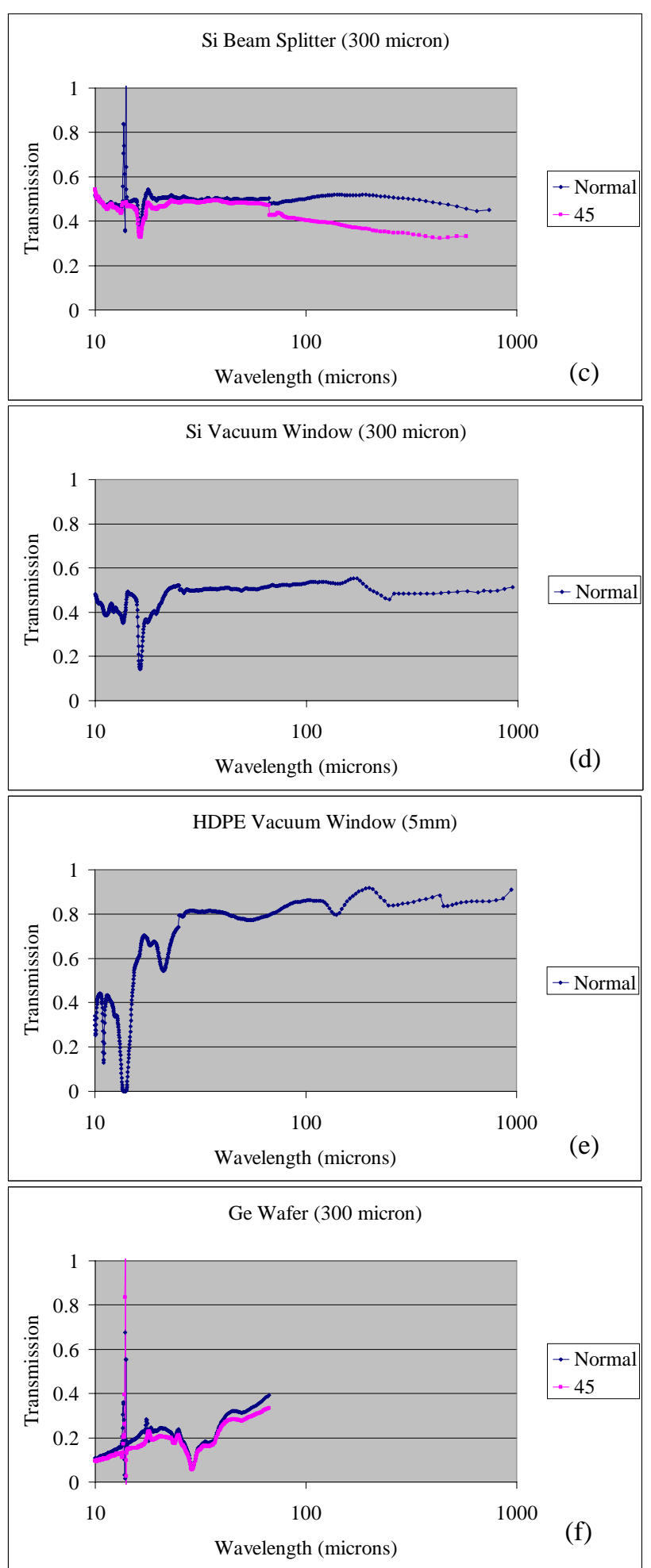

Fig. 1 Transmission spectra for common materials used as beamsplitters and vacuum windows. Data for is given for normal and 45 degree incidence for beamsplitters and normal incidence for vacuum windows.
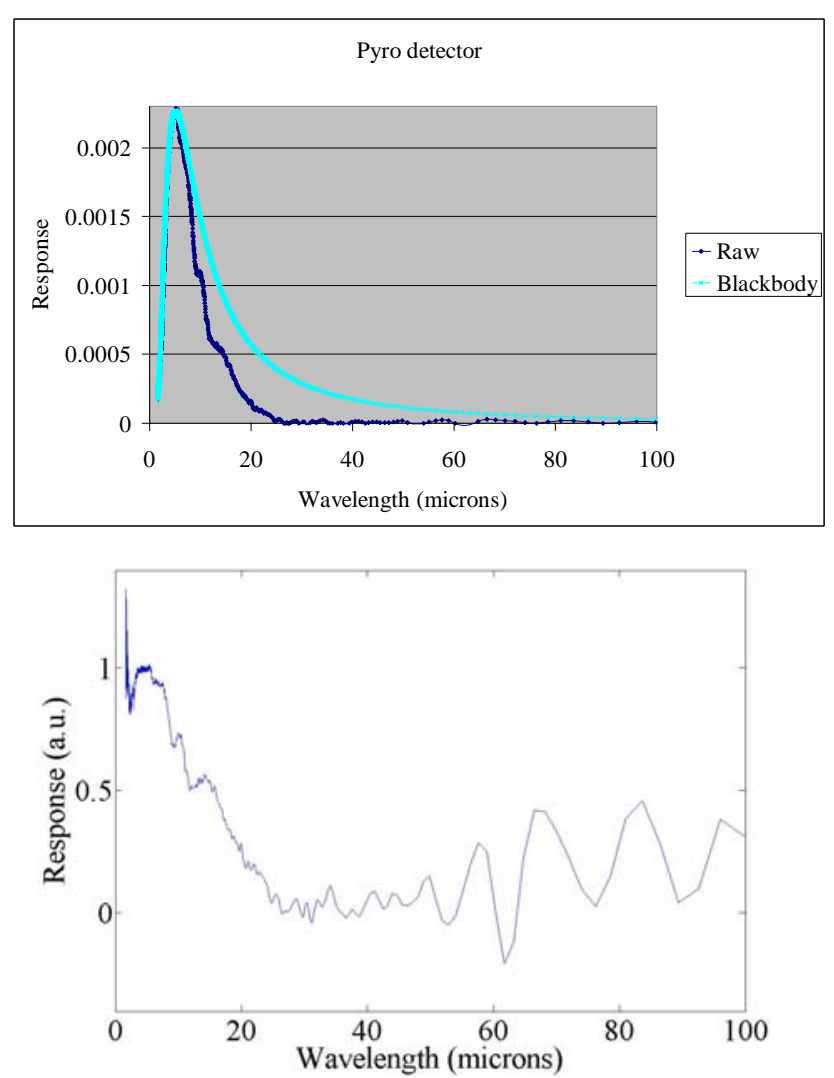

Fig. 2 P1-45 pyrodetector response. (Top) Raw, uncalibrated data with a blackbody radiation spectrum at 993K. (Bottom) Detector response vs. wavelength assuming a source blackbody emission spectra at $993 \mathrm{~K}$.

\section{DISCUSSION}

Figure 3 shows an ACT constructed to measure the electron bunch length. On the top left is the raw ACT. On the top right is the associated power spectrum. A digital filter is applied to the raw ACT to remove material effects. Due to uncertainties in the pyroelectric detector response curve, the curve was assumed constant for all frequencies. See below for further comments on the pyrodetector. The digital filter was constructed by taking the products of the Fourier transforms of the appropriate spectra shown in figure 1. Each ACT was fitted with a least squares Lorentzian curve. For this example data set, the FWHM of the filtered ACT is 25\% larger than the FWHM of the unfiltered ACT. Similar narrowing of the raw ACT with respect to the filtered ACT has been observed for bunch lengths between $23.3 \mu \mathrm{m}$ and $47.3 \mu \mathrm{m}$.

Attempts to calibrate the P1-45 pyroelectric detector in the FIR have been unsuccessful. The spectral region of interest for CTR produced from ultra-short electron bunches is between $10 \mu \mathrm{m}$ and $1,000 \mu \mathrm{m}$. The relatively low infrared output of the globar combined with the pyroelectric detector response makes calibration with this technique over the majority of the required spectral range impractical. In order to accurately calibrate the pyroelectric detector, a high power FIR source such as the LCLS should be used. 

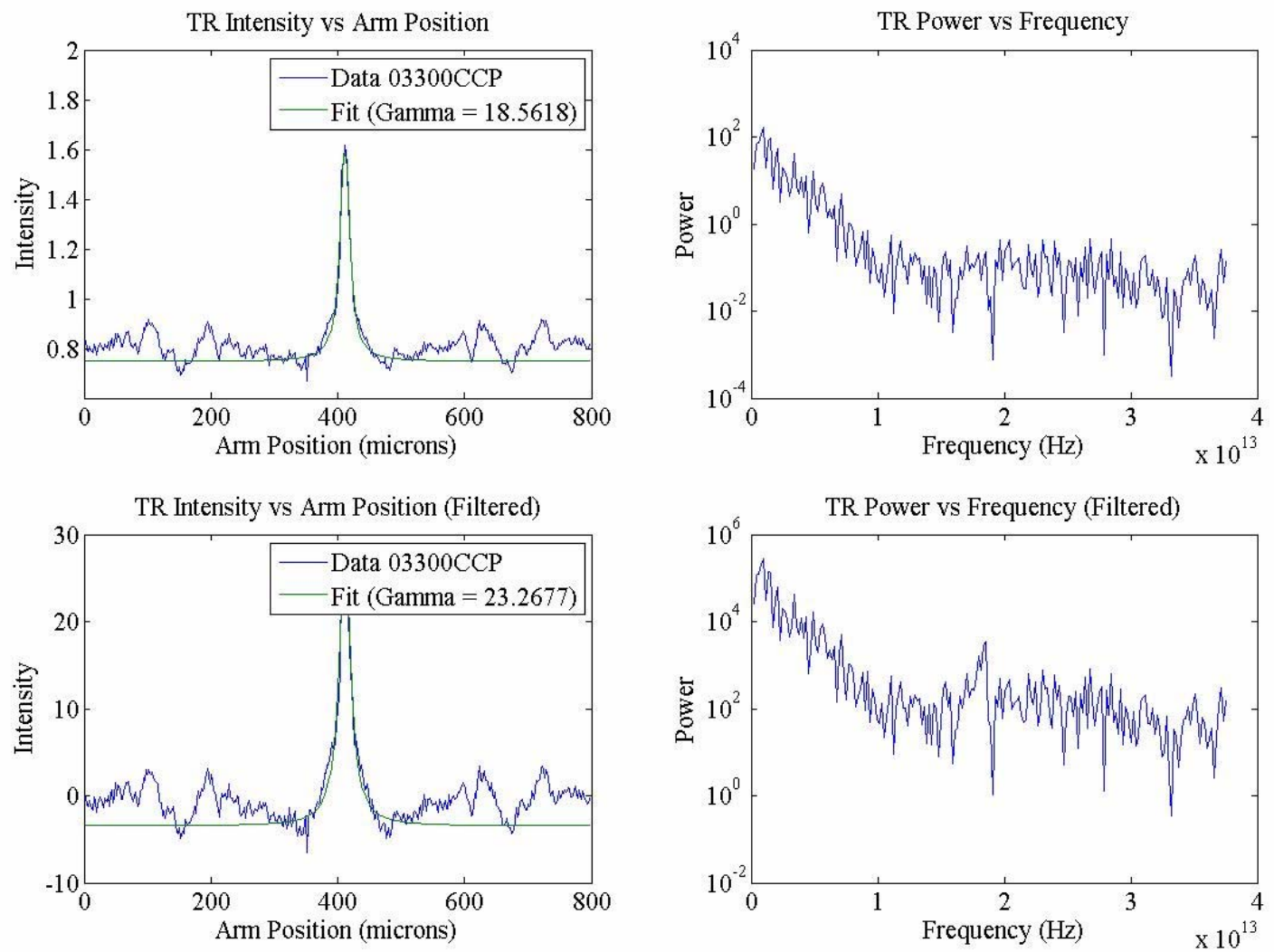

Figure 3. Sample auto-correlation trace of coherent transition radiation (CTR). (Top left) Raw Data. (Top right) Power spectrum. (Bottom left) Auto-correlation trace with corrections for material properties. (Bottom right) Corrected power spectrum. The corrected bunch length is approximately $25 \%$ larger.

\section{ACKNOWLEDGEMENTS}

The authors would like to acknowledge Michael C. Martin and Zhao Hao from the Advanced Light Source at Lawrence Berkeley National Lab for the use of the Bruker FTIR as well their personal support and assistance. 